\title{
Pulsed-Current-Induced Domain Wall Propagation in Permalloy Patterns Observed Using Magnetic Force Microscope
}

\author{
L. Gan, S. H. Chung, K. H. Aschenbach, M. Dreyer, and R. D. Gomez, Member, IEEE
}

\begin{abstract}
Pulsed-current controlled wall motion in $20 \mu \mathrm{m}$ wide $\times 200 \mu \mathrm{m}$ long $\times 160 \mathrm{~nm}$ thick patterned Permalloy strips was studied using magnetic force microscopy. By sequential imaging, the displacement of Bloch walls as far as $200 \mu \mathrm{m}$ along the strip was observed. The direction of motion was in the same direction as the carrier velocity, which reversed with current polarity. The displacement per pulse was dependent upon the sample thickness and current density, which suggests that the mechanism is a combination of s-d exchange and hydromagnetic domain drag forces.
\end{abstract}

Index Terms-Permalloy, current pulse, domains, Bloch wall, s-d exchange, domain drag, MFM, magnetization switching.

\section{INTRODUCTION}

$\mathbf{T}$ HE realization of most magnetic memory and spin-dependent devices is intricately related to the ability to change the magnetization of one or more of the active elements. A common way of switching the magnetization is by generating a magnetic "write" field higher than the coercivity of the ferromagnetic layer. However, a potential drawback for this technique is the need for a high external current to induce magnetization reversal. Further, as the critical dimensions decrease, the field localization for selective switching could become a critical concern. Thus, it is useful to explore alternative means of changing the local magnetization other than direct field generation [1]. The recently proposed VCR-voltage controlled rotation [2] is very appealing, but the fabrication requirements appear to be quite stringent. In this paper, we are interested in exploiting the well-known phenomena of domain drag induced by current pulses and applying the technique to small patterned structures similar to studies of patterned GdCoMo films [3]. The devices that could potentially benefit from these investigations are magnetic tunnel junctions [4], as well as MRAM's and spin transistors that utilize Permalloy as a spin sensing electrode. The purpose of this paper is to understand domain wall motion in patterned $100-160 \mathrm{~nm}$ thin Permalloy films. We will demonstrate the technical feasibility of moving domains with directional specificity. We provide

Manuscript received February 14, 2000. This work was supported in part by UMCP NSF-MRSEC.

L. Gan, K. H. Aschenbach, M. Dreyer, and R. D. Gomez are with Department of Electrical and Computer Engineering, University of Maryland, College Park, MD 20742 USA (e-mail: rdgomez@eng.umd.edu)

S. H. Chung is with the Department of Physics, University of Maryland, College Park, MD 20742 USA.

Publisher Item Identifier S 0018-9464(00)08511-3. some insights on the complex nature of wall motion as well as ascertain the mechanism of domain wall motion.

\section{EXPERIMENTAL TECHNIQUE}

The structures were fabricated by using conventional photolithography and lift-off. One set of patterns were $20 \mu \mathrm{m}$ wide rectangular strips with variable length from $100 \mu \mathrm{m}$ to $300 \mu \mathrm{m}$, and a second set had a "bow-tie" shape with the same interior geometry but with large flared ends. After development, the substrates were placed in a high vacuum system and evacuated at $1 \times 10^{-5}$ Torr. NiFe films were deposited on native oxide by thermal evaporation of $\mathrm{Ni}_{84} \mathrm{Fe}_{16}$ pellets. The background was $3 \times 10^{-5}$ Torr and the deposition rate was kept at $10 \AA / \mathrm{s}$. No external magnetic field was applied during deposition process. The film thickness varied from 100-160 nm. Thin $(\sim 25 \mu \mathrm{m})$ gold wires were connected on both ends of the islands using silver paint, which provided the electrical connection to our pulse generator. The DC electrical resistance of the patterns was about $40-70 \Omega$. The current pulses were generated by a $5 \mathrm{nF}$ capacitor charged up to $100 \mathrm{~V}$ and switched to discharge through the sample. The pulse shape, as monitored using a storage oscilloscope, had better than $10 \mathrm{~ns}$ rise time and an exponential decay on the order of $1 \mu \mathrm{s}$. The magnetic domains were imaged after every pulse by using a large platform magnetic force microscope. The large contact area of the bow-tie structure reduced the mechanical stress from the paint which enabled larger current densities to be applied.

\section{RESUlTS AND Discussion}

Fig. 1 shows a sequence of MFM images of the same area of the strip. Also shown is a high-resolution detail of a domain wall. The domain walls are clearly visible in all the islands, from which we can deduce the magnetization of the domains. The interior regions are comprised of alternating magnetization patterns oriented nominally at $20^{\circ}$ to the strip. The triangular features near the edges are the ubiquitous closure domains whose magnetizations are parallel to the edges to reduce magnetostatic energy. The film thickness ranged from $100 \mathrm{~nm}$ to $160 \mathrm{~nm}$ so that the walls are expected to be Bloch or asymmetric Bloch walls, rather than Néel. This is confirmed by considering the zoomed image in Fig. 1(d). The contrast changes polarity twice along the wall without crossties, which precludes the possibility of Néel type wall. Additionally, the contrast variation [5] and line profiles are consistent with those expected for $180^{\circ}$ Bloch 


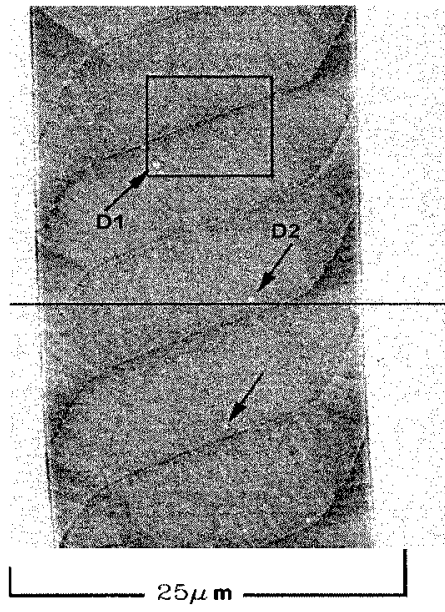

(a)

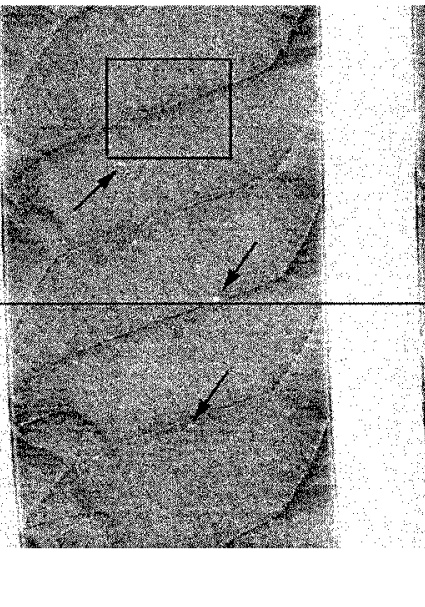

(b)

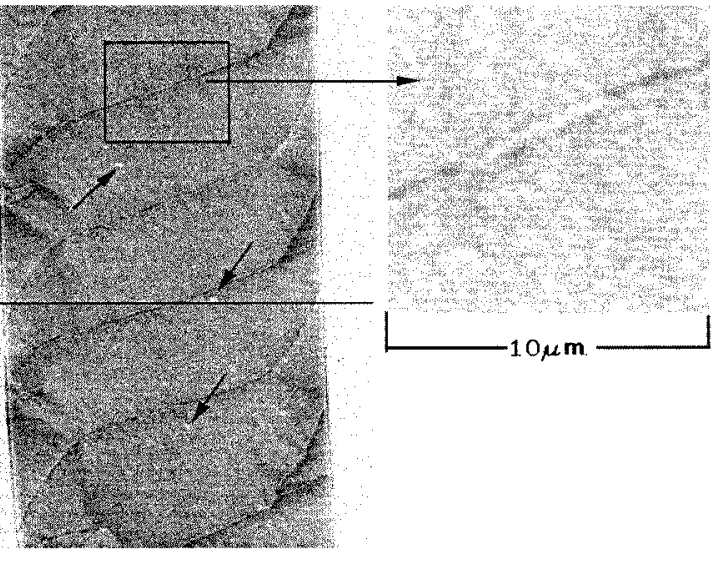

(c) (d)

Fig. 1. A sequence of magnetic domain propagation with successive current pulses. $40 \mu \mathrm{m} \times 25 \mu \mathrm{m}$ MFM scans of the same area of the surface at (a) the initial states, (b) after one pulse and (c) after two pulses. Arrows are drawn to highlight topographic defects to serve reference points for domain motion. The current direction is down and the domain walls move in the opposite direction. (d) Zoom-in-image of a segment of a Bloch wall. The boxes in (a), (b) and (c) show regions where the wall structure change with pulse.

TABLE I

\begin{tabular}{|c|c|c|c|c|c|c|}
\hline Semple & $\begin{array}{c}R \\
\text { (a) }\end{array}$ & $\begin{array}{l}\text { Volt. } \\
\text { (V) }\end{array}$ & $\begin{array}{l}\text { Thirk. } \\
(n m) \text { ) }\end{array}$ & $\begin{array}{l}\text { Width } \\
(\mu \mathrm{ma})\end{array}$ & $\begin{array}{l}\text { Cunrert } \\
\text { Density } \\
\text { a } 0^{11} \text { am }^{2}\end{array}$ & $\begin{array}{l}\text { Distance } \\
\text { (1) Pon Pube }\end{array}$ \\
\hline 0813 & 48 & 30 & 115 & 20 & 2.31 & 2.29 \\
\hline 0819 & 45 & 40 & 125 & 20 & 3.55 & 3.47 \\
\hline 0823 & 45 & 40 & 102 & 20 & 4.35 & 2.27 \\
\hline 1111 & 43 & 35 & 161 & 20 & 2.52 & 0.85 \\
\hline 1208 & 42 & 40 & 151 & 20 & 3.10 & 1.32 \\
\hline 1223 & 41 & 40 & 132 & 20 & 3.69 & 0.44 \\
\hline 0104 & 69 & 60 & 154 & 20 & 2.79 & 0.45 \\
\hline
\end{tabular}

walls [6]. Fig. 1(a)-(c) were selected from a series of numerous successive images of the same area, with one pulse applied between each consecutive image. The motion of the walls is best seen by noting the relative displacements with respect to the fixed topographical defects. As a guide to the eye, we place arrows on three topographical defects and a line is drawn to connect the middle defect of the three images. It is clear that all walls were displaced by an average distance of $0.80 \mu \mathrm{m}$ in the same direction. Moreover, the triangular edge domains themselves moved in concert with the interior walls, suggesting that the effect of the current pulse was to propagate entire domains. In this particular sample, a current density $2.5 \times 10^{11} \mathrm{~A} / \mathrm{m}^{2}$ was used and the direction of current flow was from top to bottom. Hence, the direction of the wall motion was opposite to the conventional current direction or the same as the drift velocity of the conduction electrons. More importantly, we established that the direction of domain wall motion can be reversed by switching the current polarity. These observations are true for all samples investigated, despite some differences in the critical current density.

The results from several samples are summarized in the Table I. In general, wall displacement occurs when a critical current density on the order of $10^{11} \mathrm{~A} / \mathrm{m}^{2}$ is reached. The number of samples is clearly very limited to establish the precise influence of the sample thickness and current density

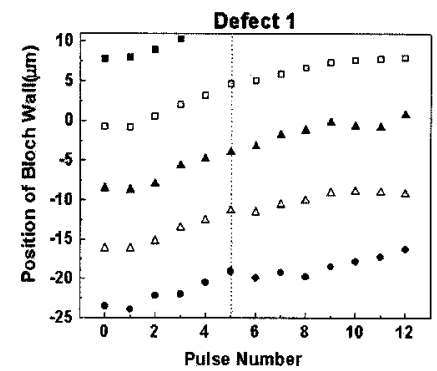

(a)

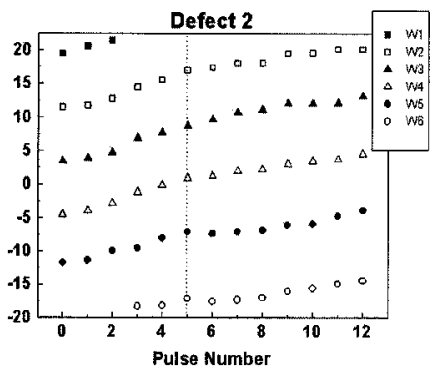

(b)
Fig. 2. The domain wall positions with respect to the defects when applying current pulses through the sample shown in Fig. 1. The top wall in the Fig. 1 is noted as W1, The second top wall as W2, so on. (a) Wall positions with respect to the left defect, (b) with respect to right defect.

on domain wall motion. However, by comparing samples 0813/1111 and 0819/1208, the trend is toward increased wall motion with decreasing sample thickness at a given current density.

Likewise, from samples 0813 and 0819 with similar thickness, higher current density leads to larger wall displacements. The motion is rather complex and does not have a simple relationship with the number of pulses. To illustrate this latter point, we plot in Fig. 2 the positions of domain walls in Fig. 1 with respect to the defects D1 and D2. The two curves show that the walls are nominally translated in the same direction. However, the domain propagation is nonlinear. This is evidenced in Fig. 2, where walls moved faster and more coherently in 2nd to 5th pulses, but were somewhat retarded in the latter pulses.

We also observed that there are specific cases when segments of the walls did not move in the same direction. An example is the movement of wall W5 after pulse 1 where the displacement measured relative to the left defect D1 is negative while the motion relative to D2 is positive. Similar events can be seen for $\mathrm{W} 4$ at pulse 6 and $\mathrm{W} 5$ at pulse 8 . These nonlinear phenomena are perhaps manifestations of the hysteretic effects of wall motion, along with pinning attributable to local variations in the 
stoichiometry and grain size. In some other samples, the domain wall motions exhibited abrupt jumps in wall motion reminiscent of Barkhausen effects [7].

Now let us address the possible mechanisms that govern the wall motion. Neglecting eddy current, viscous, pinning and other hysteretic effects, there are three major theories for magnetic domain wall displacement by current pulses. The hydromagnetic "domain drag force" is based on the Hall effect and magnetoresistance within magnetic domain [8]. The force is expected to push walls in the same sense as the conventional current if the anomalous Hall coefficient of the ferromagnetic material is positive, and vice versa. It is proportional to the sample thickness and tends to zero with very thin samples. The other mechanism is the "s-d exchange force", which is associated with the interaction of the $3 \mathrm{~d}$ electrons in the metallic ferromagnetic materials and the 4 s electrons in the conduction band of the material [9]. The domain wall displacement is always in the direction of motion of charge carriers, i.e., in the opposite sense as the conventional current. Unlike the domain drag, the exchange force is independent of the sample thickness. The third mechanism is caused by the "global field" [10]. The electric current through the sample produces a circumferential magnetic field, which runs in closed loops perpendicular to the direction of current. The magnetic field component along the loop has opposite signs at the top and bottom of the film and its magnitude is proportional to the distance from the center of cross section of the sample. The global field exerts torque on the wall causing an S-shaped distortion of a Bloch wall and makes adjacent walls move in opposite directions. Another form of global field exerts a torque on the domain walls and reduces the coercivity during oscillation of domain wall after the pulse [11]. It can thus induce ballistic wall motion after the end of the pulse with much less current density.

In our experiments, the walls show uniform motion in the same direction, contrary to the predictions of the global field theory. The critical current is also rather large, which means that the coercivity is not reduced significantly by the current pulse. This leads us to conclude that global field is not important in our case. Now we are left with distinguishing the contributions from the "hydromagnetic domain-drag" and "s-d exchange" forces. Both of these models predict motion in one direction. The direction of the wall motion due to domain drag is dependent upon the anomalous Hall coefficient, $R_{1}$. For positive $R_{1}$, the motion is in the same sense as the conventional current. At room temperature, $R_{1}$ of $\mathrm{NiFe}$ changes from negative to positive at a concentration of $15 \%$ Fe or higher [12]. Without direct measurements, we therefore can not positively conclude the sign of the anomalous Hall coefficient in our films. The only possibility for domain drag to be consistent with the sense of the observed wall motion is for $R_{1}$ to be negative. If this indeed $R_{1}$ is negative and the hydromagnetic drag is dominant, then the theory predicts that the force is proportional to the sample thickness. However, from Table I, the thinner films exhibit larger displacements for similar currents, which is opposite to the effect predicted by the domain drag dominance. Moreover, Fig. 1 shows large contrast change inside Bloch walls after each pulse, which implies some interaction between conduction electrons and valence electrons inside the domain walls. These observations suggest that the wall motion is governed by the combination of the s-d exchange and hydromagnetic drag force, which acts in opposite directions.

Using the established models [8], [9], we can calculate the critical thickness beyond which the domain drag will become dominant.

$$
w_{c}=\frac{\pi^{3} \rho \beta_{1}}{8.4 M_{s} \mu_{i}} \times \frac{R_{0}}{R_{1}}
$$

where $\rho$ and $M_{s}$ are electrical resistively and saturation magnetization of Permalloy sample, $\mu_{i}$ is the intrinsic wall mobility and $\beta_{1}$ a dimensionless correction coefficient of order unity. $R_{0}$ and $R_{1}$ are normal Hall constant and anomalous Hall constant respectively. Using the typical material data in the literature [12], [13], we find that the critical thickness is $\sim 1 \mu \mathrm{m}$ for our Permalloy sample. Establishing that the mechanism is s-d exchange is quite important for device applications. Since the force is independent of the sample thickness, it is possible and in fact easier to move domain wall for very thin samples.

\section{CONCLUSION}

These experiments clearly demonstrate the feasibility of using short current pulses as control signals to alter the magnetization of a specific area of the surface. As a rough estimate, we can realize a device $4 \mu \mathrm{m}$ wide by $10 \mu \mathrm{m}$ long by $25 \mathrm{~nm}$ thick that can exhibit a $1 \mu \mathrm{m}$ wall displacement per pulse at $3 \mathrm{~V}$ and $35 \mathrm{~mA}$.

\section{REFERENCES}

[1] E. B. Myers, D. C. Ralph, J. A. Katine, R. N. Louie, and R. A. Buhrman, Science, vol. 285, pp. 867-870, 1999.

[2] C. Y. You and S. D. Bader, J. Magn. Magn. Mater, vol. 195, pp. 488-500, 1999.

[3] J. C. DeLuca, R. J. Gambino, and A. P. Malozemoff, IEEE Trans. Magn., vol. 14 , no. 5, pp. 500-502, 1978.

[4] T. Miyazaki, S. Kumagai, and T. Yaoi, J. Appl. Phys., vol. 81, pp. 3753-3757, 1997.

[5] S. Foss, E. D. Dahlberg, R. Proksch, and B. M. Moskowitz, J. Appl. Phys., vol. 81, pp. 5032-5034, 1997.

[6] U. Hartmann, Phys. Rev. B, vol. 40, pp. 7471-7424, 1989.

[7] H. J. Williams and W. Shockley, Phys. Rev., vol. 75, pp. 178-183, 1949.

[8] W. J. Carr, J. Appl. Phys., vol. 45, pp. 394-396, 1974. S. H. Charap, J. Appl. Phys., vol. 45, pp. 397-402, 1974; L. Berger, J. Appl. Phys., vol. 49, pp. 2156-2161, 1978.

[9] L. Berger, J. Appl. Phys., vol. 55, pp. 1954-1956, 1984. L. Berger, J. Appl. Phys., vol. 71, pp. 2721-2726, 1992.

[10] C.-Y. Hung and L. Berger, J. Appl. Phys., vol. 63, pp. 4276-4278, 1988.

[11] E. Salhi and L. Berger, J. Appl. Phys., vol. 76, pp. 4787-4792, 1994. E. Salhi and L. Berger, J. Appl. Phys., vol. 73, pp. 6405-6407, 1993.

[12] J. Smit, Physica, vol. 21, pp. 877-887, 1955. W. Jellinghaus and M. P. DeAndres, Ann. Physik, vol. 5, pp. 187-199, 1960.

[13] P. P. Freitas and L. Berger, J. Appl. Phys., vol. 57, pp. 1266-1269, 1985. 